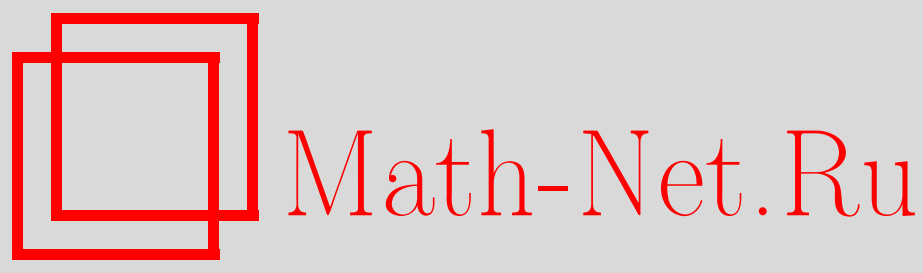

А. Я. Силенко, Оператор Гамильтона и квазиклассический предел для скалярных частиц в электромагнитном поле, ТМФ, 2008, том 156, номер 3, 398-411

DOI: https://doi.org/10.4213/tmf6255

Использование Общероссийского математического портала Math-Net.Ru подразумевает, что вы прочитали и согласны с пользовательским соглашением http://www . mathnet.ru/rus/agreement

Параметры загрузки:

IP : 18.208 .226 .222

26 апреля 2023 г., $12: 24: 42$

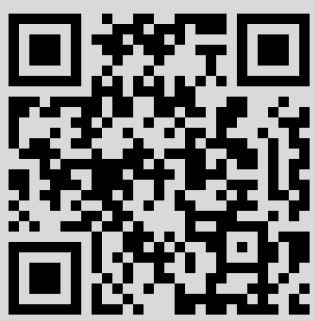




\title{
ОПЕРАТОР ГАМИЛЬТОНА И КВАЗИКЛАССИЧЕСКИЙ ПРЕДЕЛ ДЛЯ СКАЛЯРНЫХ ЧАСТИЦ В ЭЛЕКТРОМАГНИТНОМ ПОЛЕ
}

\begin{abstract}
С помощью последовательно проведенных обобщенного преобразования Кейза-Фолди-Фешбаха-Вилларса и преобразования Фолди-Ваутхойзена выведен оператор Гамильтона для релятивистских скалярных частиц в электромагнитном поле. Обобщенное преобразование Кейза-Фолди-Фешбаха-Вилларса, в отличие от оригинального преобразования, содержит произвольный параметр и может быть произведено для безмассовых частиц, что позволяет решить проблему безмассовых частиц в электромагнитном поле. Показано, что вид оператора Гамильтона в представлении Фолди-Ваутхойзена не зависит от произвольно выбираемого параметра. По сравнению с классическим гамильтонианом для точечных частиц оператор Гамильтона содержит слагаемые квантовой природы, характеризующие квадрупольное взаимодействие движущихся частиц с электрическим полем, электрическую и смешанную поляризуемости. Получены квантово-механические и квазиклассические уравнения движения массивных и безмассовых частиц в электромагнитном поле.
\end{abstract}

Ключевые слова: уравнение Клейна-Гордона, преобразование Кейза-Фолди-Фешбаха-Вилларса, преобразование Фолди-Ваутхойзена, скалярные частицы, электромагнитное взаимодействие.

\section{1. ВВЕДЕНИЕ}

Скалярные (бесспиновые) частицы не имеют собственных мультипольных моментов. Поэтому исследование их взаимодействия с внешним полем дает прекрасную возможность сравнения выводов классической и квантовой теорий. Дополнительные слагаемые в квантово-механическом гамильтониане описывают взаимодействие, имеющее квантовую природу.

Для частиц с нулевым спином во внешнем поле исходным является уравнение второго порядка - уравнение Клейна-Гордона (КГ). Однако оно менее удобно для получения информации о наблюдаемых величинах, чем релятивистское волновое уравнение для оператора Гамильтона или уравнение первого порядка Даффина-Кеммера-Петьо (ДКП) [1]-[3]. Уравнение ДКП успешно применяется для частиц со

* Институт ядерных проблем Белорусского государственного университета, Минск, Беларусь. E-mail: silenko@inp.minsk.by 
спинами 0 и 1. В настоящей работе оператор Гамильтона преобразуется к диагональному виду, характеризующему представление Фолди-Ваутхойзена (ФВ) [4]. Использование этого представления упрощает нахождение собственных и средних значений операторов и вывод квазиклассических уравнений движения (см. [5]).

Переход от уравнения второго порядка к уравнению первого порядка требует определенной осторожности. При этом часто извлекают квадратный корень из обеих частей операторного уравнения (см., например, [6]). Однако этот метод может приводить к неточностям [7]. Известными и хорошо обоснованными методами являются применение преобразования Кейза-Фолди-Фешбаха-Вилларса (КФФВ) к уравнению для оператора Гамильтона [8]-[10] и использование уравнения ДКП для частиц во внешнем поле [11]-[15].

Оператор Гамильтона для релятивистских скалярных точечных частиц может быть выведен с помощью метода, предложенного в [7] и использующего последовательно преобразования КФФВ и ФВ. Преобразование ФВ для нерелятивистских скалярных частиц в электромагнитном поле было проведено в [8], [10], [16]. В настоящей работе используется обобщенное преобразование КФФВ, которое можно применить и для безмассовых частиц. Выводится уравнение для оператора Гамильтона, описывающего взаимодействие массивных и безмассовых релятивистских скалярных частиц с электромагнитным полем.

Использована система единиц, в которой $\hbar=c=1$. В разделах 5,6 мы явно вводим постоянную $ћ$ в некоторые уравнения.

\section{2. ПЕРЕХОД К УРАВНЕНИЮ ПЕРВОГО ПОРЯДКА ДЛЯ СКАЛЯРНЫХ ЧАСТИЦ}

Исходное уравнение КГ для скалярных частиц в электромагнитном поле имеет вид

$$
\left[\left(i \frac{\partial}{\partial t}-e \varphi\right)^{2}-(\mathbf{p}-e \mathbf{A})^{2}-m^{2}\right] \psi=0
$$

где $\varphi$ и $\mathbf{A}-$ скалярный и векторный потенциалы электромагнитного поля, $\mathbf{p}=-i \nabla$, $e$ и $m$ - заряд и масса частицы, $\psi$ - однокомпонентная волновая функция. Это уравнение второго порядка. Одним из основных методов исследования взаимодействия скалярных частиц с внешним полем является переход к уравнению первого порядка относительно производной по времени. Такой переход может быть произведен с помощью преобразования КФФВ, предложенного в [8]-[10]. Его результатом является представление волнового уравнения для скалярных частиц в гамильтоновой форме.

Отметим, что подобное преобразование к уравнению первого порядка производится и для частиц со спином 1. Хотя исходные релятивистские волновые уравнения второго порядка для частиц со спинами 0 и 1 существенно различаются между собой, их преобразование имеет некоторые общие черты и производится с помощью подобных методов. Для частиц со спином 1 такое преобразование было выполнено в работе [17]. Обобщенное преобразование Сакаты-Такетани для частиц, имеющих аномальный магнитный момент и электрический квадрупольный момент, было проведено в [18]. Во всех случаях результатом преобразований является уравнение для недиагонального гамильтониана, действующего на биспинорную волновую 
функцию. Волновые функции уравнения для оператора Гамильтона, получаемого в результате преобразования КФФВ, формально также можно рассматривать как биспиноры. Для бесспиновых частиц "спиноры” однокомпонентны, а “биспинорные" волновые функции двухкомпонентны.

Хотя для частиц с целочисленным спином уравнения для недиагональных гамильтонианов формально подобны уравнению Дирака, вид оператора Гамильтона и нормировка волновой функции существенно иные, чем для частиц со спином $1 / 2$. В то же время существует определенная аналогия между свойствами оператора Гамильтона и волновых функций для частиц со спинами 0 и 1. Волновые функции частиц со спинами $0,1 / 2$ и 1 (биспиноры) могут быть записаны в общем виде

$$
\Psi=\left(\begin{array}{l}
\phi \\
\chi
\end{array}\right)
$$

где $\phi$ и $\chi-$ верхний и нижний спиноры. Нормировка волновых функций для частиц со спинами 0 и 1 имеет вид [18], [19]

$$
\int \Psi^{\dagger} \rho_{3} \Psi d V=1
$$

Здесь и ниже $\rho_{i}, i=1,2,3,-$ матрицы Паули, компоненты которых действуют на спиноры $\phi, \chi$ :

$$
\rho_{1}=\left(\begin{array}{cc}
0 & 1 \\
1 & 0
\end{array}\right), \quad \rho_{2}=\left(\begin{array}{cc}
0 & -i \\
i & 0
\end{array}\right), \quad \rho_{3} \equiv \beta=\left(\begin{array}{cc}
1 & 0 \\
0 & -1
\end{array}\right) .
$$

Гамильтониан для частиц со спинами 0 и 1 является псевдоэрмитовым, или, точнее, $\beta$-псевдоэрмитовым (см. [20] и цитированную там литературу), и неэрмитовым в обычном смысле. Он удовлетворяет соотношению [20]

$$
\mathcal{H}^{\dagger}=\beta \mathcal{H} \beta, \quad \beta^{-1}=\beta,
$$

которое эквивалентно [18], [19]

$$
\mathcal{H}^{\ddagger} \equiv \rho_{3} \mathcal{H}^{\dagger} \rho_{3}=\mathcal{H} .
$$

Определим [19] псевдоскалярное произведение двух волновых функций $\Psi_{1}$ и $\Psi_{2}$ как интеграл:

$$
\left\langle\Psi_{1} \mid \Psi_{2}\right\rangle=\int \Psi_{1}^{\dagger} \rho_{3} \Psi_{2} d V
$$

Тогда независимо от исходного представления псевдоскалярное произведение не меняется при псевдоунитарном преобразовании вида $\Psi_{1,2}^{\prime}=U \Psi_{1,2}$, оператор которого обладает следующим свойством [19]:

$$
U^{\ddagger} \equiv \rho_{3} U^{\dagger} \rho_{3}=U^{-1} .
$$

Для скалярных частиц недиагональный оператор Гамильтона, действующий на двухкомпонентную волновую функцию и полученный путем преобразования волнового уравнения второго порядка (уравнения КГ), был найден в работе Кейза [8]. 
Метод нахождения нужного преобразования был описан Фолди [9]. В настоящее время обычно используется вариант этого метода, предложенный Фешбахом и Вилларсом [10]. Поэтому мы будем называть представление, в котором задан указанный выше недиагональный оператор Гамильтона, представлением КФФВ.

$\mathrm{C}$ помощью псевдоунитарного преобразования, которым является преобразование $\Phi В$, недиагональный оператор Гамильтона можно преобразовать из исходного представления КФФВ (представления Сакаты-Такетани для частиц со спином 1) к диагональному (для частиц со спином 1 - к блочно-диагональному, т.е. диагональному по двум спинорам) виду. Для релятивистских свободных скалярных частиц и в нерелятивистском пределе для скалярных частиц в электромагнитном поле такое преобразование было выполнено в [8], [10], [16].

Переход от уравнения второго порядка КГ к уравнению первого порядка, выполненный с помощью преобразования КФФВ, является точным. Результирующее уравнение определяет недиагональный оператор Гамильтона, действующий на двухкомпонентную "биспинорную” волновую функцию (2).

В работе [9] был указан метод преобразования к уравнению первого порядка для свободных частиц путем перехода к двухкомпонентной волновой функции $\Psi$. Для частиц во внешнем поле подобное преобразование, которое в настоящее время является общепринятым, было выполнено в работе Фешбаха и Вилларса [10] (см. также [19]). Оно заключается во введении волновых функций, удовлетворяющих условиям [10], [19]

$$
\psi=\phi+\chi, \quad i \frac{\partial \psi}{\partial t}-e \varphi \psi=m(\phi-\chi)
$$

В этом случае двухкомпонентная волновая функция имеет вид

$$
\Psi=\frac{1}{2}\left(\begin{array}{c}
\psi+\frac{1}{m}\left[i \frac{\partial \psi}{\partial t}-e \varphi \psi\right] \\
\psi-\frac{1}{m}\left[i \frac{\partial \psi}{\partial t}-e \varphi \psi\right]
\end{array}\right)
$$

В работах [21] было показано, что в общем случае преобразование уравнения (1) в уравнение первого порядка производится по формулам вида (7), (8), в которых массу частицы $m$ можно заменить любым ненулевым параметром. Поскольку преобразование КФФВ [8]-[10] применимо только для массивных частиц, представляет интерес рассмотрение обобщенного преобразования [21], которое можно использовать и для безмассовых частиц. Мы будем исследовать обобщенное преобразование КФФВ, для которого масса $m$ заменена произвольной действительной ненулевой константой. Наличие произвольного параметра позволяет определенным образом менять вид обобщенного преобразования КФФВ. В результате форма промежуточного уравнения с недиагональным (по компонентам волновой функции) гамильтонианом в обобщенном представлении КФФВ также может варьироваться. Однако, как будет показано ниже, конечное выражение для оператора Гамильтона в представлении $Ф В$ не зависит от вида указанного промежуточного уравнения.

4 Теоретическая и математическая физика, т. 156, № 3, 2008 г. 
Используемое в настоящей работе обобщенное преобразование применимо и для безмассовых частиц. Это обстоятельство является весьма важным, поскольку уравнения для безмассовых частиц не могут быть получены из уравнений для массивных частиц путем перехода к пределу $m \rightarrow 0$ (см. [22] и цитированную там литературу).

Таким образом, при подходе, который применяется в настоящей работе, волновые функции $\phi$ и $\chi$ определяются уравнениями

$$
\psi=\phi+\chi, \quad\left(i \frac{\partial}{\partial t}-e \varphi\right) \psi=N(\phi-\chi),
$$

где $N$ - произвольный действительный ненулевой параметр.

Если умножить последнее уравнение на множитель $i \partial / \partial t-e \varphi$, то уравнения (9) могут быть представлены в матричной форме:

$$
i \frac{\partial \Psi}{\partial t}=\mathcal{H} \Psi, \quad \mathcal{H}=\rho_{3} \frac{\boldsymbol{\pi}^{2}+m^{2}+N^{2}}{2 N}+e \varphi+i \rho_{2} \frac{\boldsymbol{\pi}^{2}+m^{2}-N^{2}}{2 N},
$$

где $\boldsymbol{\pi}=\mathbf{p}-e \mathbf{A}=-i \nabla-e \mathbf{A}-$ оператор кинетического импульса, $\rho_{i}-$ матрицы Паули (3), компоненты которых действуют на соответствующие компоненты волновой функции $\Psi$.

Уравнение, эквивалентное (10), рассмотрено в [21]. В работе Фешбаха и Вилларса [10] рассмотрен частный вид этого уравнения при $N=m$.

\section{3. ПРЕОБРАЗОВАНИЕ ФОЛДИ-ВАУТХОЙЗЕНА ДЛЯ РЕЛЯТИВИСТСКИХ ЧАСТИЦ ВО ВНЕШНЕМ ПОЛЕ}

Представление ФВ, описанное в классической работе [4], занимает особое место в релятивистской квантовой механике. В этом представлении соотношения между операторами полностью аналогичны соотношениям между соответствующими классическими величинами. Операторы в представлении ФВ имеют такой же вид, как в нерелятивистской квантовой механике. Весьма важно, что в данном представлении очень простой вид имеют операторы координат $\mathbf{r}$ и импульса $\mathbf{p}=-i \nabla$. Именно представление ФВ обеспечивает наилучшую возможность получения классического предела релятивистской квантовой механики [4], [5], [23].

Результатом преобразования волновой функции к данному представлению (преобразования ФВ) является переход к квазидиагональной (диагональной по двум спинорам) форме гамильтониана. При этом происходит разделение состояний с положительной и отрицательной полной энергией. В представлении ФВ не возникает проблемы "дрожания" (Zitterbewegung). Преобразование ФВ радикально упрощает переход к квазиклассическому пределу, для которого, в частности, не требуется такая процедура, как выделение четных частей операторов.

Мы используем преобразование $Ф В$ в одночастичном приближении, когда радиационные поправки не рассчитываются с помощью теоретико-полевых методов, а учитываются феноменологически путем включения дополнительных слагаемых в релятивистские волновые уравнения (подобно аномальному магнитному моменту [24]). Одночастичное приближение, естественно, применимо и для релятивистских частиц, когда при заданной энергии взаимодействия вероятностью рождения пар и потерями на тормозное излучение можно пренебречь. 
Гамильтониан $\mathcal{H}$ можно разложить на операторы, коммутирующие и антикоммутирующие с оператором $\rho_{3}$ :

$$
\mathcal{H}=\rho_{3} \mathcal{M}+\mathcal{E}+\mathcal{O}, \quad \rho_{3} \mathcal{M}=\mathcal{M} \rho_{3}, \quad \rho_{3} \mathcal{E}=\mathcal{E} \rho_{3}, \quad \rho_{3} \mathcal{O}=-\mathcal{O} \rho_{3}
$$

В рассматриваемом случае

$$
\mathcal{M}=\frac{\pi^{2}+m^{2}+N^{2}}{2 N}, \quad \mathcal{E}=e \varphi, \quad \mathcal{O}=i \rho_{2} \frac{\pi^{2}+m^{2}-N^{2}}{2 N} .
$$

Очень важно, что уравнения, определяющие оператор Гамильтона для частиц со спинами $0,1 / 2$ и 1 , имеют один и тот же вид (11). Поэтому формально совпадают и соответствующие выражения для оператора $U$, приводящего оператор Гамильтона к блочно-диагональному виду. Это позволяет применять для скалярных частиц методы преобразования $\Phi В$, разработанные для частиц со спинами 1/2 [4], [5], [25]-[29] и 1 [30], [31]. Однако следует учитывать, что, как было впервые показано в [32], не любая диагонализация гамильтониана приводит к представлению ФВ. Для примера можно взять преобразование Эриксена-Корлсруда [33], приводящее оператор Гамильтона к блочно-диагональному виду. В работе [34] было доказано, что даже для свободных частиц оно не приводит к представлению ФВ.

Существует ряд методов преобразования ФВ для нерелятивистских частиц со спином $1 / 2$, позволяющих рассчитать релятивистские поправки [4], [25], [27]-[29]. Классический метод такого преобразования, предложенный в [4] (см. также [27]) для случая $\mathcal{M}=m$, заключается в применении оператора

$$
U=e^{i S}, \quad S=-\frac{i}{2 m} \rho_{3} \mathcal{O} .
$$

Преобразованный гамильтониан может быть записан в виде

$$
\begin{aligned}
\mathcal{H}^{\prime}=\mathcal{H} & +i[S, \mathcal{H}]+\frac{i^{2}}{2 !}[S,[S, \mathcal{H}]]+\frac{i^{3}}{3 !}[S,[S,[S, \mathcal{H}]]]+\cdots- \\
& -\dot{S}-\frac{i}{2 !}[S, \dot{S}]-\frac{i^{2}}{3 !}[S,[S, \dot{S}]]-\cdots
\end{aligned}
$$

где $[\cdot, \cdot]$ - коммутатор. В результате преобразования (14) гамильтониан определяется уравнением [4], [27]

$$
\mathcal{H}^{\prime}=\rho_{3} \epsilon+\mathcal{E}^{\prime}+\mathcal{O}^{\prime}, \quad \rho_{3} \mathcal{E}^{\prime}=\mathcal{E}^{\prime} \rho_{3}, \quad \rho_{3} \mathcal{O}^{\prime}=-\mathcal{O}^{\prime} \rho_{3},
$$

в котором $\epsilon=\sqrt{m^{2}+p^{2}}$, а нечетный оператор $\mathcal{O}^{\prime}$ теперь имеет порядок $O(1 / m)$. Для достижения требуемой точности это преобразование может повторяться многократно.

Однако для релятивистских частиц во внешнем поле переход к представлению ФВ в общем случае достаточно сложен. Есть серьезные аргументы [32] в пользу того, что для дираковских частиц в произвольном внешнем поле точное решение этой задачи получено Эриксеном [25]. Однако найденное в [25] выражение содержит квадратные корни из матричных операторов, что, как правило, не позволяет 
получить явный вид оператора Гамильтона. Для релятивистских частиц его также весьма трудно представить в виде ряда по степеням энергии взаимодействия с внешним полем. Ввиду сложности указанное точное решение не использовалось при проведении преобразования ФВ для релятивистских частиц во внешнем поле. В настоящей работе такое преобразование выполнено при помощи метода, разработанного в работе [5] для частиц со спином $1 / 2$. Этот метод позволяет найти релятивистский гамильтониан в виде ряда по степеням потенциалов внешнего поля и их производных. В некоторых частных случаях данный метод приводит к точному преобразованию ФВ [5].

Для нерелятивистских частиц со спином 1 в электромагнитном поле преобразование ФВ было выполнено в [18], а в релятивистском случае - в [30], [31]. Преобразование ФВ для нерелятивистских скалярных частиц в электромагнитном поле было использовано в [8], [10], [16], а для релятивистских - в [7]. В работе [16] также было выполнено нерелятивистское преобразование ФВ для системы двух частиц: бозона со спином 0 и фермиона со спином $1 / 2$.

Хотя исходные релятивистские волновые уравнения для частиц со спинами 0 и 1 существенно различаются между собой, их преобразование к представлению ФВ имеет общие черты и производится с помощью подобных методов. Прежде всего общей чертой является необходимость проведения предварительного преобразования, приводящего исходные уравнения к гамильтоновой форме. Это преобразования КФФВ [8]--[10] для скалярных частиц и Сакаты-Такетани [17] для частиц со спином 1. Обобщенное преобразование Сакаты-Такетани для частиц, имеющих аномальный магнитный момент и электрический квадрупольный момент, было выполнено в [18]. Во всех случаях результатом является уравнение для недиагонального гамильтониана, действующего на биспинорную волновую функцию.

При выполнении условий коммутации

$$
[\mathcal{M}, \mathcal{O}]=0, \quad[\mathcal{E}, \mathcal{O}]=0
$$

и в стационарном внешнем поле может быть произведено точное преобразование ФВ. В этом случае гамильтониан $\mathcal{H}$ преобразуется к блочно-диагональному виду с помощью оператора [5], [31]:

$$
U=\frac{\epsilon+\mathcal{M}+\rho_{3} \mathcal{O}}{\sqrt{2 \epsilon(\epsilon+\mathcal{M})}}, \quad U^{-1}=\frac{\epsilon+\mathcal{M}-\rho_{3} \mathcal{O}}{\sqrt{2 \epsilon(\epsilon+\mathcal{M})}}, \quad \epsilon=\sqrt{\mathcal{M}^{2}+\mathcal{O}^{2}} .
$$

Трансформированный гамильтониан имеет вид [5], [31]

$$
\mathcal{H}^{\prime}=\rho_{3} \epsilon+\mathcal{E}
$$

В общем случае, определяемом формулами (11), (12), внешнее поле нестационарно, оператор $\mathcal{O}$ коммутирует с $\mathcal{M}$, но может не коммутировать с $\mathcal{E}$. Мы проводим расчеты в приближении слабого поля и полагаем, что энергия взаимодействия мала по сравнению с полной энергией, включающей энергию покоя $m c^{2}$ и приблизительно равной $\epsilon$. При использовании приближения слабого поля малыми безразмерными параметрами, по которым производится разложение, являются отношения тех слагаемых в операторе взаимодействия частицы с внешним полем, которые пропорциональны первым степеням потенциалов поля и их пространственных и временных 
производных, к полной энергии частицы. Полное решение задачи дает выражение для оператора Гамильтона в представлении $Ф В$ в виде ряда по степеням потенциалов внешнего поля и их производных.

Используемый нами метод, разработанный в [5], [31], заключается в следующем. Сначала производится псевдоунитарное преобразование с оператором (17) (см. [5], [31]). После этого преобразования гамильтониан $\mathcal{H}^{\prime}$ еще содержит нечетные слагаемые, пропорциональные производным от потенциалов, и может быть записан в виде

$$
\mathcal{H}^{\prime}=\rho_{3} \epsilon+\mathcal{E}^{\prime}+\mathcal{O}^{\prime}, \quad \rho_{3} \mathcal{E}^{\prime}=\mathcal{E}^{\prime} \rho_{3}, \quad \rho_{3} \mathcal{O}^{\prime}=-\mathcal{O}^{\prime} \rho_{3}
$$

где (см. [5])

$$
\begin{gathered}
\mathcal{E}^{\prime}=\mathcal{E}-\frac{1}{4}\left[\frac{\epsilon+\mathcal{M}}{\sqrt{\epsilon(\epsilon+\mathcal{M})}},\left[\frac{\epsilon+\mathcal{M}}{\sqrt{\epsilon(\epsilon+\mathcal{M})}}, \mathcal{F}\right]\right]+ \\
+\frac{1}{4}\left[\frac{\beta \mathcal{O}}{\sqrt{\epsilon(\epsilon+\mathcal{M})}},\left[\frac{\beta \mathcal{O}}{\sqrt{\epsilon(\epsilon+\mathcal{M})}}, \mathcal{F}\right]\right] \\
\mathcal{O}^{\prime}=\frac{\beta \mathcal{O}}{\sqrt{2 \epsilon(\epsilon+\mathcal{M})}} \mathcal{F} \frac{\epsilon+\mathcal{M}}{\sqrt{2 \epsilon(\epsilon+\mathcal{M})}}-\frac{\epsilon+\mathcal{M}}{\sqrt{2 \epsilon(\epsilon+\mathcal{M})}} \mathcal{F} \frac{\beta \mathcal{O}}{\sqrt{2 \epsilon(\epsilon+\mathcal{M})}}, \\
\mathcal{F}=\mathcal{E}-i \frac{\partial}{\partial t}
\end{gathered}
$$

$\epsilon$ определяется формулой (17). При использовании приближения слабого поля нечетный оператор $\mathcal{O}^{\prime}$ мал по сравнению как с $\epsilon$, так и с исходным гамильтонианом $\mathcal{H}$. Это позволяет использовать на второй стадии обычную схему, характерную для нерелятивистского преобразования ФВ [4], [5], [27], [31]. Такое преобразование выполняется с помощью оператора

$$
U^{\prime}=e^{i S^{\prime}}, \quad S^{\prime}=-\frac{i}{4} \rho_{3}\left\{\mathcal{O}^{\prime}, \frac{1}{\epsilon}\right\}=-\frac{i}{4}\left[\frac{\rho_{3}}{\epsilon}, \mathcal{O}^{\prime}\right],
$$

где $\{\cdot, \cdot\}$ - антикоммутатор. Дальнейшие вычисления такие же, как в случае частиц со спинами $1 / 2$ и 1 (см. [5], [27], [31]). В отличие от [27], в данном случае масса частицы должна быть заменена на оператор $\epsilon$, не коммутирующий с операторами $\mathcal{E}^{\prime}$ и $\mathcal{O}^{\prime}$. Если учитывать только основные поправки, которые пропорциональны $\mathcal{O}^{\prime 2}$, т.е. вторым степеням потенциалов поля и их пространственных и временных производных, то преобразованный гамильтониан приобретает вид [5]

$$
\mathcal{H}_{\mathrm{F} W}=\mathcal{H}^{\prime \prime}=\rho_{3} \epsilon+\mathcal{E}^{\prime}+\frac{\rho_{3}}{4}\left\{\frac{1}{\epsilon}, \mathcal{O}^{\prime 2}\right\} .
$$

Для достижения требуемой точности процедура преобразования с оператором (21) ( $S^{\prime}$ заменяется на $S^{\prime \prime}, S^{\prime \prime \prime}$ и т.д.) может повторяться многократно.

\section{4. ПРЕОБРАЗОВАНИЕ ФОЛДИ-ВАУТХОЙЗЕНА ДЛЯ СКАЛЯРНЫХ ЧАСТИЦ}

Проведем преобразование ФВ для релятивистских скалярных частиц в электромагнитном поле с помощью описанного выше метода. Мы полагаем, что энергия 
взаимодействия мала по сравнению с полной энергией, включающей энергию покоя. Для гамильтониана, определяемого формулами (11), (12),

$$
\epsilon=\sqrt{m^{2}+\pi^{2}}
$$

а псевдоунитарный оператор преобразования (17) в рассматриваемом случае может быть приведен к виду

$$
U=\frac{\epsilon+N+\rho_{1}(\epsilon-N)}{2 \sqrt{\epsilon N}} .
$$

В результате преобразования исходного гамильтониана, определяемого формулами (11), (12), с помощью оператора (24) трансформированный гамильтониан $\mathcal{H}^{\prime}$ еще содержит нечетные слагаемые и имеет вид (19), где

$$
\mathcal{E}^{\prime}=i \frac{\partial}{\partial t}+\frac{1}{2}\left(\sqrt{\epsilon} \mathcal{F} \frac{1}{\sqrt{\epsilon}}+\frac{1}{\sqrt{\epsilon}} \mathcal{F} \sqrt{\epsilon}\right), \quad \mathcal{O}^{\prime}=\frac{\rho_{1}}{2}\left(\sqrt{\epsilon} \mathcal{F} \frac{1}{\sqrt{\epsilon}}-\frac{1}{\sqrt{\epsilon}} \mathcal{F} \sqrt{\epsilon}\right)
$$

а $\epsilon$ определяется формулой (23). Используя соотношения коммутации, формулы (25) можно преобразовать к виду

$$
\mathcal{E}^{\prime}=\mathcal{E}+\frac{1}{2 \sqrt{\epsilon}}[\sqrt{\epsilon},[\sqrt{\epsilon}, \mathcal{F}]] \frac{1}{\sqrt{\epsilon}}, \quad \mathcal{O}^{\prime}=\rho_{1} \frac{1}{2 \sqrt{\epsilon}}[\epsilon, \mathcal{F}] \frac{1}{\sqrt{\epsilon}} .
$$

Формулы (23), (25), (26) являются точными для произвольного оператора $\mathcal{E}$ и не зависят от $N$. Это означает, что оператор Гамильтона в представлении $\Phi$ В, получаемый в результате следующего этапа преобразований, также не зависит от $N$. Представление КФФВ таким свойством не обладает. Таким образом, последовательное проведение обобщенного преобразования КФФВ и преобразования ФВ еще раз демонстрирует особую роль представления $\Phi В$ в физике частиц, на этот раз на примере частиц со спином 0.

Приближенно (см. общие формулы для коммутаторов в [5])

$$
[\epsilon, \mathcal{F}]=\frac{1}{4}\left\{\frac{1}{\epsilon},\left[\boldsymbol{\pi}^{2}, \mathcal{F}\right]\right\}, \quad[\sqrt{\epsilon},[\sqrt{\epsilon}, \mathcal{F}]]=\frac{1}{32}\left\{\frac{1}{\epsilon^{3}},\left[\boldsymbol{\pi}^{2},\left[\boldsymbol{\pi}^{2}, \mathcal{F}\right]\right]\right\} .
$$

Оператор Гамильтона в представлении $\Phi B$, который может быть найден по приближенной формуле (22), равен

$$
\mathcal{H}_{\mathrm{FW}}=\rho_{3} \epsilon+\mathcal{E}+\frac{1}{64}\left\{\frac{1}{\epsilon^{4}},\left[\boldsymbol{\pi}^{2},\left[\boldsymbol{\pi}^{2}, \mathcal{F}\right]\right]\right\}+\frac{\rho_{3}}{64}\left\{\frac{1}{\epsilon^{5}},\left(\left[\boldsymbol{\pi}^{2}, \mathcal{F}\right]\right)^{2}\right\} .
$$

Проведенные вычисления носят общий характер, и формулы (23)-(27) справедливы в случае взаимодействия скалярных частиц с любым внешним полем, гамильтониан которого определяется уравнениями $(11),(12)$ с произвольными $\mathcal{E}$ и $\boldsymbol{\pi}$. Для электромагнитного взаимодействия, описываемого формулами (11), (12),

$$
\begin{aligned}
& {\left[\boldsymbol{\pi}^{2}, \mathcal{F}\right]=2 i e \boldsymbol{\pi} \cdot \mathbf{E}, \quad } {\left[\boldsymbol{\pi}^{2},\left[\boldsymbol{\pi}^{2}, \mathcal{F}\right]\right]=4 e(\boldsymbol{\pi} \cdot \nabla)(\boldsymbol{\pi} \cdot \mathbf{E})-4 e^{2} \boldsymbol{\pi} \cdot(\mathbf{E} \times \mathbf{H}), } \\
&(\boldsymbol{\pi} \cdot \nabla)(\boldsymbol{\pi} \cdot \mathbf{E}) \equiv \pi_{i} \pi_{j} \frac{\partial E_{j}}{\partial x_{i}} .
\end{aligned}
$$


В результате находим окончательное приближенное выражение для оператора Гамильтона в представлении $Ф В$, характеризующего взаимодействие точечных скалярных частиц с электромагнитным полем:

$$
\mathcal{H}_{\mathrm{F} W}=\rho_{3} \epsilon+e \varphi+\frac{e}{8 \epsilon^{4}}(\boldsymbol{\pi} \cdot \nabla)(\boldsymbol{\pi} \cdot \mathbf{E})-\frac{e^{2}}{8 \epsilon^{4}} \boldsymbol{\pi} \cdot(\mathbf{E} \times \mathbf{H})-\rho_{3} \frac{e^{2}}{8 \epsilon^{5}}(\boldsymbol{\pi} \cdot \mathbf{E})^{2} .
$$

Отметим, что последовательное проведение обобщенного преобразования КФФВ и преобразования ФВ позволило вывести оператор Гамильтона, который описывает как массивные, так и безмассовые частицы, и, таким образом, решить проблему безмассовых частиц в электромагнитном поле. Оригинальное преобразование [8]-[10], как следует из уравнений (7), (8), не может проводиться при $m=0$. При использовании оригинального метода [8]-[10] случай безмассовых частиц не может быть рассмотрен как предельный при $m \rightarrow 0$ для случая массивных частиц, поскольку при таком подходе масса является множителем, что делает нахождение предела при $m \rightarrow 0$ бесполезным. Точно такая же ситуация имеет место при попытке использования данного предела для описания безмассовых частиц с помощью уравнения ДКП (см. [22]).

В формулах (28), (29) мы не учитываем порядок операторов, имея в виду последующий переход к квазиклассическому приближению. Такой переход требует соблюдения условия малости средних значений коммутаторов операторов динамических переменных (координат и импульса) по сравнению со средними значениями произведений этих операторов. В этом случае можно переставлять соответствующие некоммутирующие операторы в квантово-механических выражениях.

\section{5. СРАВНЕНИЕ СВОЙСТВ ЧАСТИЦ В КЛАССИЧЕСКОЙ И КВАНТОВОЙ ЭЛЕКТРОДИНАМИКЕ}

Квазиклассический переход может быть выполнен аналогично работе [5]. Указанное выше условие малости средних значений коммутаторов операторов координат и импульса по сравнению со средними значениями произведений этих операторов автоматически выполняется в том случае, когда характерный размер области неоднородности внешнего поля значительно больше, чем длина волны де Бройля частицы: $l \gg \hbar / p$. В этом случае квазиклассический переход производится путем тривиальной замены операторов в квантово-механических уравнениях для верхнего спинора соответствующими классическими величинами.

Полученный квазиклассический гамильтониан имеет вид

$$
\mathcal{H}_{\mathrm{s}}=\epsilon+e \varphi+\frac{e}{8 \epsilon^{4}}(\boldsymbol{\pi} \cdot \nabla)(\boldsymbol{\pi} \cdot \mathbf{E})-\frac{e^{2}}{8 \epsilon^{4}} \boldsymbol{\pi} \cdot(\mathbf{E} \times \mathbf{H})-\frac{e^{2}}{8 \epsilon^{5}}(\boldsymbol{\pi} \cdot \mathbf{E})^{2} .
$$

Соответствующий классический гамильтониан содержит только два первых слагаемых:

$$
\mathcal{H}_{\mathrm{c}}=\epsilon+e \varphi \text {. }
$$

Хотя частицы предполагаются точечными, три последних слагаемых в квазиклассическом гамильтониане (29) описывают свойства, характерные для составных классических частиц. Однако гамильтониан не содержит слагаемого, характеризующего 
контактное (дарвиновское) взаимодействие покоящихся частиц. Более того, для покоящихся частиц все квантовые поправки обращаются в нуль. Это свойство согласуется с результатами, полученными в работе [35]. Приведенные в этой работе вывод оператора Гамильтона для частиц произвольного спина и последующее преобразование ФВ для нерелятивистских частиц показывают, что появление статического контактного (дарвиновского) взаимодействия связано с наличием у частиц спина. Отсутствие такого взаимодействия для частиц с нулевым спином проявляется в том, что слагаемое, характеризующее контактное взаимодействие [35], обращается в бесконечность при $S=0$.

Включение постоянной $\hbar$ в уравнение (29) показывает, что три последних слагаемых пропорциональны $\hbar^{2}$ :

$$
\mathcal{H}_{\mathrm{s}}=\epsilon+e \varphi+\frac{e \hbar^{2}}{8 \epsilon^{4}}(\boldsymbol{\pi} \cdot \nabla)(\boldsymbol{\pi} \cdot \mathbf{E})-\frac{e^{2} \hbar^{2}}{8 \epsilon^{4}} \boldsymbol{\pi} \cdot(\mathbf{E} \times \mathbf{H})-\frac{e^{2} \hbar^{2}}{8 \epsilon^{5}}(\boldsymbol{\pi} \cdot \mathbf{E})^{2} .
$$

Движущиеся частицы имеют ряд неклассических свойств. Третье слагаемое в (29) описывает квадрупольное взаимодействие с электрическим полем. Аналогичное слагаемое входит в гамильтониан для частиц со спином 1/2 [5], [26]. Последнее слагаемое характеризует электрическую поляризуемость движущихся частиц, которая также не равна нулю для точечных частиц со спином 1/2 [26], а предыдущее слагаемое - смешанную поляризуемость. Индуцированный электрический дипольный момент равен

$$
\mathbf{d}=\frac{\partial \mathcal{H}_{\mathrm{s}}}{\partial \mathbf{E}}=\frac{e^{2} \hbar^{2}}{8 \epsilon^{4}} \boldsymbol{\pi} \times \mathbf{H}-\frac{e^{2} \hbar^{2}}{4 \epsilon^{5}} \boldsymbol{\pi}(\boldsymbol{\pi} \cdot \mathbf{E}) .
$$

Индуцированный магнитный дипольный момент определяется формулой

$$
\boldsymbol{\mu}=\frac{\partial \mathcal{H}_{\mathrm{s}}}{\partial \mathbf{H}}=-\frac{e^{2} \hbar^{2}}{8 \epsilon^{4}} \boldsymbol{\pi} \times \mathbf{E} .
$$

Таким образом, индуцированный электрический дипольный момент пропорционален напряженности магнитного поля, а индуцированный магнитный дипольный момент пропорционален напряженности электрического поля. Эти свойства имеют квантовую природу.

Квантово-механическое уравнение движения частицы имеет вид

$$
\frac{d \boldsymbol{\pi}}{d t}=\frac{i}{\hbar}\left[\mathcal{H}_{\mathrm{F} W}, \boldsymbol{\pi}\right]-e \frac{\partial \mathbf{A}}{\partial t} .
$$

В рассматриваемом случае его квазиклассический предел определяется формулой

$$
\begin{gathered}
\frac{d \boldsymbol{\pi}}{d t}=e \mathbf{E}+\frac{e}{\epsilon}(\boldsymbol{\pi} \times \mathbf{H})+\frac{e^{2} \hbar^{2}}{8 \epsilon^{4}}[(\boldsymbol{\pi} \cdot \nabla)(\mathbf{E} \times \mathbf{H})-(\mathbf{H} \times \nabla)(\boldsymbol{\pi} \cdot \mathbf{E})]+ \\
+\frac{e^{3} \hbar^{2}}{8 \epsilon^{4}}\left[H^{2} \mathbf{E}-\mathbf{H}(\mathbf{E} \cdot \mathbf{H})\right]-\frac{e^{3} \hbar^{2}}{4 \epsilon^{5}}(\boldsymbol{\pi} \cdot \mathbf{E})(\mathbf{E} \times \mathbf{H})
\end{gathered}
$$

Квантовые поправки к классическому выражению, определяющему силу Лоренца, очень малы. 


\section{6. УРАВНЕНИЕ ДАФФИНА-КЕММЕРА-ПЕТЬО ДЛЯ ЧАСТИЦ В ЭЛЕКТРОМАГНИТНОМ ПОЛЕ И ПРОБЛЕМА БЕЗМАССОВЫХ ЧАСТИЦ}

Уравнение ДКП [1]-[3] для частиц со спинами 0 и 1 является аналогом уравнения Дирака. Для свободных скалярных частиц оно имеет вид

$$
\left(\beta^{\mu} \partial_{\mu}-m\right) \Phi=0
$$

где

$$
\begin{aligned}
& \beta^{0}=\left(\begin{array}{ccccc}
0 & 0 & 0 & 0 & -1 \\
0 & 0 & 0 & 0 & 0 \\
0 & 0 & 0 & 0 & 0 \\
0 & 0 & 0 & 0 & 0 \\
1 & 0 & 0 & 0 & 0
\end{array}\right), \quad \beta^{1}=\left(\begin{array}{ccccc}
0 & 0 & 0 & 0 & 0 \\
0 & 0 & 0 & 0 & 1 \\
0 & 0 & 0 & 0 & 0 \\
0 & 0 & 0 & 0 & 0 \\
0 & 1 & 0 & 0 & 0
\end{array}\right), \\
& \beta^{2}=\left(\begin{array}{ccccc}
0 & 0 & 0 & 0 & 0 \\
0 & 0 & 0 & 0 & 0 \\
0 & 0 & 0 & 0 & 1 \\
0 & 0 & 0 & 0 & 0 \\
0 & 0 & 1 & 0 & 0
\end{array}\right), \quad \beta^{3}=\left(\begin{array}{ccccc}
0 & 0 & 0 & 0 & 0 \\
0 & 0 & 0 & 0 & 0 \\
0 & 0 & 0 & 0 & 0 \\
0 & 0 & 0 & 0 & 1 \\
0 & 0 & 0 & 1 & 0
\end{array}\right)
\end{aligned}
$$

Ковариантное обобщение этого уравнения для частиц со спином 0 в электромагнитном поле принимает вид [11]-[13]

$$
\left(\beta^{\mu} D_{\mu}-m\right) \Phi=0, \quad D_{\mu}=\partial_{\mu}+i e A_{\mu}
$$

где $A_{\mu}=(\varphi,-\mathbf{A})$ - четырехмерный потенциал электромагнитного поля. Запишем уравнение $(39)$ в явном виде:

$$
\begin{aligned}
& -D_{0} \Phi_{5}=m \Phi_{1}, \quad D_{1} \Phi_{5}=m \Phi_{2}, \quad D_{2} \Phi_{5}=m \Phi_{3}, \quad D_{3} \Phi_{5}=m \Phi_{4}, \\
& D_{0} \Phi_{1}+D_{1} \Phi_{2}+D_{2} \Phi_{3}+D_{3} \Phi_{4}=m \Phi_{5} .
\end{aligned}
$$

Эквивалентность уравнений ДКП и КГ доказана не только для электромагнитного [11]-[13], но и для других взаимодействий [12], [13], [15]. Исключение компонент $\Phi_{2}, \Phi_{3}, \Phi_{4}$ из уравнения (40) приводит к соотношениям

$$
-D_{0} \Phi_{5}=m \Phi_{1}, \quad D_{0} \Phi_{1}=\left(m-\frac{D_{1}^{2}+D_{2}^{2}+D_{3}^{2}}{m}\right) \Phi_{5} .
$$

Преобразование $\Phi_{1}=\phi-\chi, \Phi_{5}=-i(\phi+\chi)$ позволяет получить уравнение

$$
i D_{0} \Psi=\left[\rho_{3}\left(m-\frac{D_{1}^{2}+D_{2}^{2}+D_{3}^{2}}{2 m}\right)-i \rho_{2} \frac{D_{1}^{2}+D_{2}^{2}+D_{3}^{2}}{2 m}\right] \Psi, \quad \Psi=\left(\begin{array}{c}
\phi \\
\chi
\end{array}\right),
$$

которое эквивалентно уравнению (10) при условии, что $N=m$. Исключение $\Phi_{1}$ из уравнения (41) дает исходное уравнение (1).

Однако уравнение ДКП (39) неприменимо к безмассовым частицам даже при переходе к пределу $m \rightarrow 0$ [22], в то время как предложенный в настоящей работе 
метод, базирующийся на обобщенном преобразовании КФФВ, работает и в этом случае. Модификация уравнения ДКП, позволяющая описать свободные безмассовые частицы, была найдена в [22]. Релятивистское волновое уравнение для оператора Гамильтона в представлении ФВ дает исчерпывающее квантово-механическое описание взаимодействия безмассовых частиц с электромагнитным полем. Естественно, оно не учитывает квантово-полевых эффектов. Для безмассовых частиц данное уравнение в соответствии с формулой (29) для гамильтониана имеет вид

$$
i \hbar \frac{\partial \Psi_{\mathrm{F} W}}{\partial t}=\left[\rho_{3} \epsilon+e \varphi+\frac{e \hbar^{2}}{8 c^{2} \epsilon^{2}} \frac{\partial E_{x}}{\partial x}-\frac{e^{2} \hbar^{2}}{8 c \epsilon^{3}}(\mathbf{E} \times \mathbf{H})_{x}-\rho_{3} \frac{e^{2} \hbar^{2}}{8 c^{2} \epsilon^{3}} E_{x}^{2}\right] \Psi_{\mathrm{F} W},
$$

где $\epsilon=c \sqrt{\boldsymbol{\pi}^{2}}$, а направление движения частицы $\mathbf{l}=c \boldsymbol{\pi} / \epsilon$ выбрано в качестве оси $x$. В представлении $Ф \mathrm{~B}$ можно использовать только верхний спинор, поскольку нижний спинор, характеризующий состояния с отрицательной полной энергией, для реальных частиц равен нулю. В этом случае релятивистское волновое уравнение приобретает вид

$$
i \hbar \frac{\partial \phi_{\mathrm{F} W}}{\partial t}=\left[\epsilon+e \varphi+\frac{e \hbar^{2}}{8 c^{2} \epsilon^{2}} \frac{\partial E_{x}}{\partial x}-\frac{e^{2} \hbar^{2}}{8 c \epsilon^{3}}(\mathbf{E} \times \mathbf{H})_{x}-\frac{e^{2} \hbar^{2}}{8 c^{2} \epsilon^{3}} E_{x}^{2}\right] \phi_{\mathrm{F} W} .
$$

Уравнения (29), (43), (44) (как и исходное уравнение КГ) эквивалентны уравнению ДКП, но их отличительной чертой является то обстоятельство, что квантовые поправки к классическому гамильтониану представлены в явном виде. Эти поправки имеют релятивистский характер и обращаются в нуль в статическом пределе. Подобные поправки появляются и в квантово-механическом гамильтониане для частиц со спином $1 / 2$ [5], [26].

\section{7. ВЫВОДЫ}

В настоящей работе исходное уравнение КГ для скалярных частиц в электромагнитном поле приведено к гамильтоновой форме с помощью последовательно проведенных обобщенного преобразования КФФВ и преобразования ФВ. Выведены релятивистские формулы для оператора Гамильтона и квазиклассического гамильтониана. Обобщенное преобразование КФФВ, в отличие от оригинального преобразования, содержит произвольный параметр, что позволяет использовать его для безмассовых частиц. Показано, что вид оператора Гамильтона в представлении ФВ не зависит от произвольно выбираемого параметра. Решена проблема безмассовых частиц в электромагнитном поле. По сравнению с классическим гамильтонианом для точечных частиц оператор Гамильтона и квазиклассический гамильтониан содержат слагаемые квантовой природы, характеризующие квадрупольное взаимодействие движущихся частиц с электрическим полем, электрическую и смешанную поляризуемости. Эти слагаемые имеют релятивистский характер и обращаются в нуль в статическом пределе. Подобные квантовые поправки к классическому гамильтониану появляются и для частиц со спином $1 / 2$. Получены квантово-механические и квазиклассические уравнения движения массивных и безмассовых частиц в электромагнитном поле. Произведено сравнение найденных уравнений с уравнением ДКП. 
Благодарности. Работа поддержана грантом Белорусского республиканского фонда фундаментальных исследований.

\section{Список литературы}

[1] R. J. Duffin, Phys. Rev. (2), 54:12 (1938), 1114-1114.

[2] N. Kemmer, Proc. Roy. Soc. Edinburgh Sect. A, 173:952 (1939), 91-116.

[3] G. Petiau, Contribution a la theorie des equations d'ondes corpusculaires, Acad. Roy. Belg. Cl. Sci. Mem. Collect. $8^{\circ}$ (3), 16, 1936.

[4] L. L. Foldy, S. A. Wouthuysen, Phys. Rev. (2), 78:1 (1950), 29-36.

[5] A. J. Silenko, J. Math. Phys., 44:7 (2003), 2952-2966.

[6] A. Accioly, H. Blas, Phys. Rev. D, 66:6 (2002), 067501; Modern Phys. Lett. A, 18:12 (2003), $867-873$.

[7] А. Я. Силенко, ЯФ, 64:5 (2001), 1048-1053.

[8] K. M. Case, Phys. Rev. (2), 95:5 (1954), 1323-1328.

[9] L. L. Foldy, Phys. Rev. (2), 102:2 (1956), 568-581.

[10] H. Feshbach, F. Villars, Rev. Modern Phys., 30:1 (1958), 24-45.

[11] M. Nowakowski, Phys. Lett. A, 244:5 (1998), 329-337.

[12] Б. М. Пиментел, В. Я. Файнберг, ТМФ, 124:3 (2000), 445-462.

[13] V. Ya. Fainberg, B. M. Pimentel, Phys. Lett. A, 271:1-2 (2000), 16-25.

[14] I. V. Kanatchikov, Rept. Math. Phys., 46:1-2 (2000), 107-112.

[15] J. T. Lunardi, B. M. Pimentel, R. G. Teixeira, "Duffin-Kemmer-Petiau equation in Riemannian space-times", Geometrical Aspects of Quantum Fields (Londrina, 2000), eds. A. A. Bytsenko, A. E. Golcalves, B. M. Pimentel, World Scientific, Singapore, 2000, 111-127.

[16] T. Tanaka, A. Suzuki, M. Kimura, Z. Phys. A, 353:1 (1995), 79-85.

[17] M. Taketani, S. Sakata, Proc. Phys. Math. Soc. Japan, 22 (1940), 757-770; Progr. Theoret. Phys., 1:Suppl. 1 (1955), 84-97.

[18] J. A. Young, S. A. Bludman, Phys. Rev., 131:5 (1963), 2326-2334.

[19] А.С. Давыдов, Квантовая механика, Физматгиз, М., 1963.

[20] A. Mostafazadeh, Czechoslovak J. Phys., 53:11 (2003), 1079-1084.

[21] A. Mostafazadeh, J. Phys. A, 31:38 (1998), 7829-7845; Ann. Phys., 309:1 (2004), 1-48.

[22] R. Casana, M. Pazetti, B. M. Pimentel, J.S. Valverde, Pseudoclassical mechanics for the spin 0 and 1 particles, arXiv: hep-th/0506193, 2005.

[23] J. P. Costella, B. H. J. McKellar, Amer. J. Phys., 63:12 (1995), 1119-1121.

[24] W. Pauli, Rev. Modern Phys., 13:3 (1941), 203-232.

[25] E. Eriksen, Phys. Rev. (2), 111:3 (1958), 1011-1016.

[26] E. I. Blount, Phys. Rev. (2), 128:5 (1962), 2454-2458.

[27] Дж. Д. Бьёркен, С. Д. Дрелл, Релятивистская квантовая теория. Т. 1. Релятивистская квантовая механика, Наука, М., 1978.

[28] А.И. Ахиезер, В.Б. Берестецкий, Квантовая электродинамика, 3-е изд., Наука, М., 1969.

[29] J. G. Körner, G. Thompson, Phys. Lett. B, 264:1-2 (1991), 185-192.

[30] А.Я. Силенко, ЖЭТФ, 123:5 (2003), 883-890.

[31] A. J. Silenko, Analysis of wave equations for spin-1 particles interacting with an electromagnetic field, arXiv: hep-th/0404074, 2004.

[32] E. de Vries, J. E. Jonker, Nucl. Phys. B, 6:3 (1968), 213-225.

[33] E. Eriksen, M. Korlsrud, Nuovo Cimento (10), 18:Suppl. 1 (1960), 1-39.

[34] A. J. Silenko, O. V. Teryaev, Phys. Rev. D, 71:6 (2005), 064016.

[35] А. Г. Никитин, В. И. Фущич, ТМФ, 34:3 (1978), 319-333.

Поступила в редакцию 12.10.2005, после доработки 1.12.2007 